\title{
On the relation between the probabilistic characterization of the common cause and Bell's notion of local causality
}

\author{
Gábor Hofer-Szabó*
}

\begin{abstract}
In this paper the relation between the standard probabilistic characterization of the common cause (used for the derivation of the Bell inequalities) and Bell's notion of local causality will be investigated in the isotone net framework borrowed from algebraic quantum field theory. The logical role of two components in Bell's definition will be scrutinized; namely that the common cause is localized in the intersection of the past of the correlated events; and that it provides a complete specification of the 'beables' of this intersection.
\end{abstract}

Key words: local causality, common cause, isotone net, Bell inequality

\section{Introduction}

Standard derivations of the Bell inequalities start from a set of equations representing a probabilistic common causal explanation of correlations. This common causal explanation has three components: a screening-off condition, going back to Reichenbach's (1956) original characterization of the common cause, a locality condition, expressing probabilistic independences between spacelike separated measurement outcomes and measurement settings, and a no-conspiracy condition representing another independency between the common cause and the measurement settings. If one is asked what justifies these probabilistic constraints in representing a proper common causal explanation, the common answer is this: one obtains these equations immediately if one endorses special relativity and looks at the spacetime localization of the events in question. The aim of this paper is to understand more thoroughly this quick answer.

In order to see more clearly how the spatiotemporal and probabilistic characterization of the common cause relate to one another, one has to be clear first of all on three points:

1. To address the problem at all, we need to have a mathematically well-defined and physically wellmotivated framework connecting events understood as elements of a probability space and regions understood as subsets of a spacetime.

2. Having such a firm framework connecting spatiotemporal and probabilistic entities, we need to localize events, among them common causes, in the spacetime.

3. Finally, we have to be clear on what we mean under "justification of the probabilistic common causal explanation on spatiotemporal grounds".

${ }^{*}$ Institute of Philosophy, Research Center for the Humanities, Budapest, email: szabo.gabor@btk.mta.hu, Tel/Fax: +36 $1224-6778 / 84$. 
Here we briefly comment on the above three points in turn.

Ad 1. Concerning the framework, interestingly enough, there is not a wide choice of mathematical structures representing this highly important connection between probabilistic and spatiotemporal entities. Discounting one approach (Henson, 2005; commented on in the Conclusion and discussion), we are aware of only one such structure, the isotone net structure used in algebraic quantum field theory (AQFT). In AQFT observables are represented by $\left(C^{*}\right.$-)algebras associated to bounded regions of a spacetime. This association is called a net. A state $\phi$ is defined as a normalized positive linear functional on the quasilocal algebra $\mathcal{A}$ which is the inductive limit of the net. From our perspective, the two important axioms of the net are isotony and local primitive causality. Isotony requires that if a region $V_{1}$ is contained in another region $V_{2}$, then the local algebra $\mathcal{A}\left(V_{1}\right)$ associated to $V_{1}$ is a (unital $C^{*}$-)subalgebra of $\mathcal{A}\left(V_{2}\right)$. Local primitive causality is the requirement that for any region $V, \mathcal{A}(V)=\mathcal{A}\left(V^{\prime \prime}\right)$, where $V^{\prime \prime}$ is the causal completion (shadow) of $V$. The framework of isotone nets seems to be flexible enough to be used also for our purposes. The nets which we will use in this paper will be classical nets generated by local $\sigma$-subalgebras of a Boolean $\sigma$-algebra $\Sigma$. Thus we borrow a useful mathematical technique from AQFT without endorsing the operational ontology thereof.

Ad 2. Having a neat framework in hand, next we have to localize events. The localization of measurement outcomes and measurement settings is fairly straightforward, but where should we localize common causes? Obviously, the common cause is an event $C$ happening somewhere in the past of two correlated events, say $A$ and $B$. But in which past? Relativistically two spacelike separated events can have (at least) two different pasts. Let $V_{A}$ and $V_{B}$ denote the regions where $A$ and $B$, respectively are localized. One can then define the weak past of $A$ and $B$ as $\mathcal{P}^{W}\left(V_{A}, V_{B}\right):=I_{-}\left(V_{A}\right) \cup I_{-}\left(V_{B}\right)$ and the strong past of $A$ and $B$ as $\mathcal{P}^{S}\left(V_{A}, V_{B}\right):=I_{-}\left(V_{A}\right) \cap I_{-}\left(V_{B}\right)$ where $I_{-}(V)$ denotes the union of the causal pasts $I_{-}(x)$ of every point $x$ in $V$. Let us call the appropriate common causes weak and strong common causes, respectively (see Fig. 1).
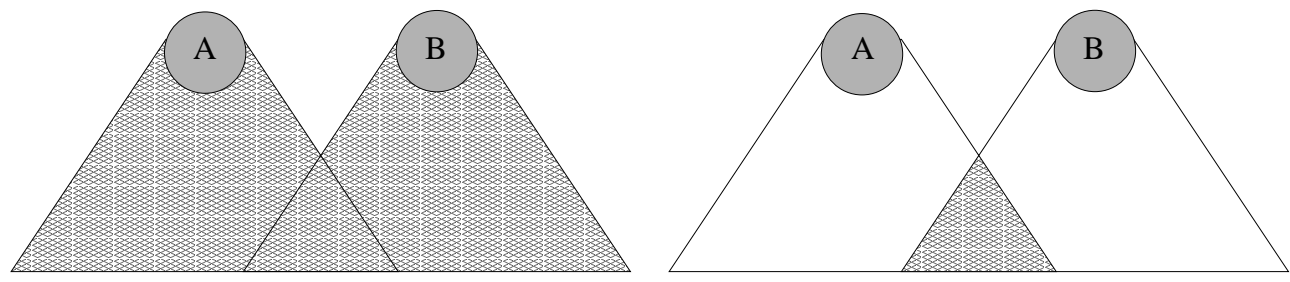

Figure 1: Weak and the strong past of the correlated events $A$ and $B$.

Now, one might consider the strong past as a more natural candidate for the localization of the common cause, and indeed plenty of classical examples attest that the strong past is a reasonable choice. The correlation between two fans' shouting at the same time at a football match is explained by the goals scored, that is by events localized in the strong past of the shouts. Curiously enough, however, in AQFT common causes are typically understood as weak common causes. It is not difficult to see why.

Consider an isotone net representing a system in AQFT. Suppose that there is a (superluminal) correlation, $\phi(A B) \neq \phi(A) \phi(B)$, between events $A \in \mathcal{A}\left(V_{A}\right)$ and $B \in \mathcal{A}\left(V_{B}\right)$ such that $V_{A}$ and $V_{B}$ are spacelike separated. Consider the local algebra $\mathcal{A}\left(\left(V_{A} \cup V_{B}\right)^{\prime \prime}\right)$ associated to the causal completion of $V_{A} \cup V_{B}$ and suppose that we find a common cause $C$ of the correlation in $\mathcal{A}\left(\left(V_{A} \cup V_{B}\right)^{\prime \prime}\right)$. In which past of $V_{A}$ and $V_{B}$ can $C$ be located? Consider a region $V$ in the weak past $P^{W}\left(V_{A}, V_{B}\right)$ which is 'wide' enough to ensure that $\left(V_{A} \cup V_{B}\right) \subset V^{\prime \prime}$. Due to isotony, $\mathcal{A}\left(V_{A} \cup V_{B}\right)$ will be a subalgebra of $\mathcal{A}\left(V^{\prime \prime}\right)$ which, due to local primitive causality, is identical to $\mathcal{A}(V)$. Thus, $C$ will be located in $V$ and hence in the weak past of $V_{A}$ and $V_{B}$. To sum up, isotony and local primitive causality together ensures that if a superluminal correlation has a common cause, then it can be localized in the weak past. 
Can the common cause be localized also in the strong past? It might, but if so, this will not be simply due to the axioms of AQFT. If $V$ is in $P^{S}\left(V_{A}, V_{B}\right)$, then isotony and local primitive causality does not help to relate $\mathcal{A}(V)$ to $\mathcal{A}\left(\left(V_{A} \cup V_{B}\right)^{\prime \prime}\right)$. One also needs to know about the dynamics of the system. The axioms of AQFT are completely silent about whether one can locate the common cause in the strong past. As a consequence, weak common causes cannot be excluded a priori from our explanatory arsenal. Thus, we had better open leave the question regarding the apt spacetime localization of the common cause.

Ad 3. Finally, we have to pin down the meaning of the term "justification of the probabilistic common causal explanation on spatiotemporal grounds". What we mean here is this: we need to have a principle regulating the probabilistic independences of events on the basis of their possible causal connectedness in tune with special relativity. An analogy for such a regulating principle might help. The theory of Bayesian nets involves two parts: a causal graph representing the causal relations among certain events and a probability space with random variables. How are these two parts of the theory related to one another? The bridge relating the two components is called the Causal Markov Condition. It says that if the nodes on the graph are related to one another in such-and-such a way, then the variables pertaining to the nodes should satisfy such-and-such probabilistic independences. So the role of the Causal Markov Condition in the theory of Bayesian nets is to coordinate the probabilistic and the graphical description of causal relations.

A principle playing a similar coordinating role in the causal explanation of correlations has been introduced into the literature by John S. Bell $(1975 / 2004)$ and called local causality. Local causality is a relativistic principle tailor-made to study probabilistic relations between events localized in different spacetime regions, among them the relation between the common cause and the correlated events. Thus, we will understand the term "justification of the probabilistic common causal explanation on spatiotemporal grounds" similarly to the Bayesian net theorist: local causality implies just those probabilistic independences which characterize the standard common causal explanation.

Putting Points 1-3 together we are faced with the following

Project. Given the isotone net framework connecting events and spacetime regions (Point 1), and given the spatiotemporal localization of the various measurement outcomes, measurement settings and common causes (Point 2), one is to define local causality in the isotone net framework such that the probabilistic independences implied by local causality (Point 3) are just the ones used in the standard probabilistic characterization of the common causal explanation.

In brief, the accommodation of a set of correlations within a locally causal net implies that for any correlations there exist common causes satisfying certain probabilistic constraints.

This, however, is only the coarse-grained story of the paper. Reading Bell's careful formulation of local causality, two requirements will stand out in the definition: one is atomicity representing the "complete specification" of the causal past of the correlated events, the other is the localization of the common cause in the strong past. Our fine-grained story will be to analyze the significance of these ingredients in the definition of local causality. It will turn out that the link between the spatiotemporal and the probabilistic characterization of the common cause is very sensitive to these components of the definition of local causality, as was rightly emphasized by Bell himself. In detail, we would like to address the following questions:

(i) What is the exact role of atomicity in the justification of the probabilistic characterization of the common cause by local causality?

(ii) Do the probabilistic constraints imposed on the notion of common cause restrict the possible spacetime localization of the common cause? Do we need to choose, for example, between weak and strong common causes? 
(iii) How do atomicity and localization relate to one another; which of the common causes localized in different pasts need to be atomic?

Our paper follows a research line which has been followed by many. To our knowledge, the first to "survey the ways in which one could associate regions" with events such that it makes "plausible not only completeness and locality, but other assumptions of the Bell inequality" was Butterfield (1989, p. 135). Also, the necessity to introduce spatiotemporal concepts so as to understand the Common Cause Principle was pointed out by Uffink (1999). Common Cause Principle and its role in the EPR-Bell scenario has been thoroughly investigated by The Bern group (Grasshoff, Portmann and Wüthrich, 2005), The Cracow group (Placek and Wronski, 2009), and The Budapest group (Hofer-Szabó, Rédei and Szabó, 2013, especially in Chapter 8 and 9). The status of the Common Cause Principle in AQFT was first investigated by Rédei (1997), and further analyzed in Poincaré covariant AQFT by Rédei and Summers (2002) and in lattice AQFT by Hofer-Szabó and Vecsernyés (2012a, 2013a). Butterfield analysed the assumptions leading to the Bell inequalities in AQFT in (Butterfield, 1995), and the relation of the Common Cause Principe to the Bell inequalities and to various forms of Stochastic Einstein Locality in (Butterfield, 2007). For an earlier discussion on the relation of Stochastic Einstein Locality to the axioms of AQFT, see (Rédei 1991) and (Muller and Butterfield 1994). Hofer-Szabó and Vecsernyés (2012b, $2013 \mathrm{~b}$ ) reassessed the assumptions of the Bell inequalities in AQFT with respect to non-commuting common causes. In a formalism very close or maybe identical to our isotone net formalism, Henson (2013b) treated an important topic, namely that giving up separability does not block the derivation of the Bell inequalities. An interesting debate between Henson, Rédei and San Pedro (Henson, 2005; Rédei and San Pedro, 2012; Henson, 2013a) has been taking place recently in this Journal. We will comment on this debate in the Conclusion and discussions. For a parallel approach to ours, where the assumptions of the Bell inequalities are backed not by spatiotemporal considerations but by the Causal Markov Condition, see (Glymour 2006). For the relation of Causal Markov Condition to EPR correlations see (Suárez, 2013). For a general treatment of Bell's local causality in local physical theories see the more technical (Hofer-Szabó and Vecsernyés 2014a) or its philosopher-friendly version (Hofer-Szabó and Vecsernyés 2014b).

Our paper is structured as follows. In Section 2 the standard requirements of the probabilistic common causal explanation will be recalled. In Section 3 Bell's original idea of local causality will be delineated and redefined in the isotone net formalism. Section 4 will be devoted to the first ingredient of Bell's definition, namely atomicity; Section 5 to the second one, namely localization. In order to proceed in a more picturesque way, both in Section 4 and 5 classical toy models will be introduced helping us to explicate the more abstract results. We conclude the paper in Section 6. Some technicalities are put in the Appendices.

\section{Common causal explanation}

As mentioned above, the first probabilistic characterization of the common cause is due to Reichenbach. There is a long route leading from Reichenbach's original idea of the common cause to the sophisticated probabilistic requirements used today in the philosophy of quantum physics. Here we will not detail the steps of how the notion of common cause evolved and became more and more suitable for causal explanation of the EPR-Bell scenario (for this see (Hofer-Szabó, Rédei and Szabó, 2013), or for a short version (Hofer-Szabó and Vecsernyés, 2012a)). Instead we will jump directly to the full-fledged probabilistic characterization of the common cause and give a brief motivation of the requirements thereafter.

Let $\left\{a_{m}\right\}$ and $\left\{b_{n}\right\}(m \in M, n \in N)$ be two sets of measurement procedures (thought of as happening in two spacelike separated spacetime regions). Suppose that each measurement can have two outcomes and

denote the 'positive' outcomes by $A_{m}$ and $B_{n}$ and the 'negative' outcomes by $\bar{A}_{m}$ and $\bar{B}_{n}$, respectively. Let all these events be accommodated in a classical probability space $(\Sigma, p)$. Suppose that there is a 
conditional correlation between the measurement outcomes in the sense that for any $m \in M$ and $n \in N$

$$
p\left(A_{m} \wedge B_{n} \mid a_{m} \wedge b_{n}\right) \neq p\left(A_{m} \mid a_{m}\right) p\left(B_{n} \mid b_{n}\right)
$$

representing that if we measure the pair $a_{m}$ and $b_{n}$, the appropriate outcomes will be correlated.

The standard probabilistic characterization of a common causal explanation of the correlations (1) is the following. A partition $\left\{C_{k}\right\}$ in $\Sigma$ (that is a set of mutually exclusive events adding up to the unit) is said to be a local, non-conspiratorial joint common causal explanation of the correlations (1) if for any $m, m^{\prime} \in M$ and $n, n^{\prime} \in N$ the following requirements hold:

$$
\begin{aligned}
p\left(A_{m} \wedge B_{n} \mid a_{m} \wedge b_{n} \wedge C_{k}\right)=p\left(A_{m} \mid a_{m} \wedge b_{n} \wedge C_{k}\right) p\left(B_{n} \mid a_{m} \wedge b_{n} \wedge C_{k}\right) & \text { (screening-off) } \\
p\left(A_{m} \mid a_{m} \wedge b_{n} \wedge C_{k}\right)=p\left(A_{m} \mid a_{m} \wedge b_{n^{\prime}} \wedge C_{k}\right) & \text { (locality) } \\
p\left(B_{n} \mid a_{m} \wedge b_{n} \wedge C_{k}\right)=p\left(B_{n} \mid a_{m^{\prime}} \wedge b_{n} \wedge C_{k}\right) & \text { (locality) } \\
p\left(a_{m} \wedge b_{n} \wedge C_{k}\right)=p\left(a_{m} \wedge b_{n}\right) p\left(C_{k}\right) & \text { (no-conspiracy) }
\end{aligned}
$$

The motivation behind requirements (2)-(5) is the following. Screening-off (2) (also called as outcome independence (Shimony, 1986), completeness (Jarrett, 1984) and causality (Van Fraassen, 1982)) is simply the application of Reichenbach's original characterization of the common cause as a screener-off to conditional correlations: although $A_{m}$ and $B_{n}$ are correlated when conditioned on $a_{m}$ and $b_{n}$, they will cease to be so, if we further condition on $C_{k}$. Locality (3)-(4) (also called as parameter independence (Shimony, 1986), locality (Jarrett, 1984) and hidden locality (Van Fraassen, 1982)) is the constraints that the measurement outcome on the one side can depend only on the measurement choice on the same side and the value of the common cause, but not on the measurement choice on the opposite side (for more on this, see below). Finally, no-conspiracy (5) is the requirement that the common cause system and the measurement settings should not influence each other: they should be probabilistically independent.

Now, it is a well known fact that if a set of correlations has a local, non-conspiratorial joint common causal explanation in the above sense, then the set of correlations has to satisfy various Bell inequalities. ${ }^{1}$ If quantum correlations are interpreted as classical conditional correlations a la (1), these Bell inequalities are violated, excluding a local, non-conspiratorial joint common causal explanation of the EPR scenario. Our aim, however, is not to follow the route leading from the common causal explanation (2)-(5) to the Bell inequalities, but rather the route leading to the common causal explanation itself. At any rate, in the EPR-Bell literature (2)-(5) is regarded as the correct probabilistic characterization of the common cause. But observe that the above motivations for the probabilistic independence relations (2)-(5) are completely meaningless unless we first decide on Points 1 and 2 of the Introduction: that is unless we have a principled way to associate events understood as elements of the probability space $(\Sigma, p)$ to regions of a given spacetime (Point 1), and unless we localize the events in question somewhere in the spacetime (Point 2).

So suppose that we do have such an association in form of an isotone net $\mathfrak{N}$ associating bounded regions of the Minkowski spacetime to $\sigma$-subalgebras of $\Sigma$. Suppose furthermore that we localize common causes in one of the two above mentioned ways, that is common causes are either weak or strong common causes. To address Point 3 of the Introduction, namely the 'bridge law' between the spacetime and probabilistic considerations, we have to introduce one more notion, namely local causality. We do this in Section 3.

\section{Local causality}

As mentioned in the Introduction, there is an influential tradition according to which equations (2)-(5) are consequences of the requirement that a certain set of correlations are to be accommodated in a locally causal theory. The clearest formulation of such a theory is due to Bell himself:

\footnotetext{
${ }^{1}$ For the derivation of one of the simplest Bell inequality, the Clauser-Horne inequality, see Appendix A.
} 
"Consider a theory in which the assignment of values to some beables $\Lambda$ implies, not necessarily a particular value, but a probability distribution, for another beable $A$. Let $p(A \mid \Lambda) \operatorname{denote}^{2}$ the probability of a particular value $A$ given particular values $\Lambda$. Let $A$ be localized in a space-time region A. Let $B$ be a second beable localized in a second region $\mathrm{B}$ separated from $\mathrm{A}$ in a spacelike way. (Fig. 2.) Now my intuitive notion of local causality is that events in $\mathrm{B}$

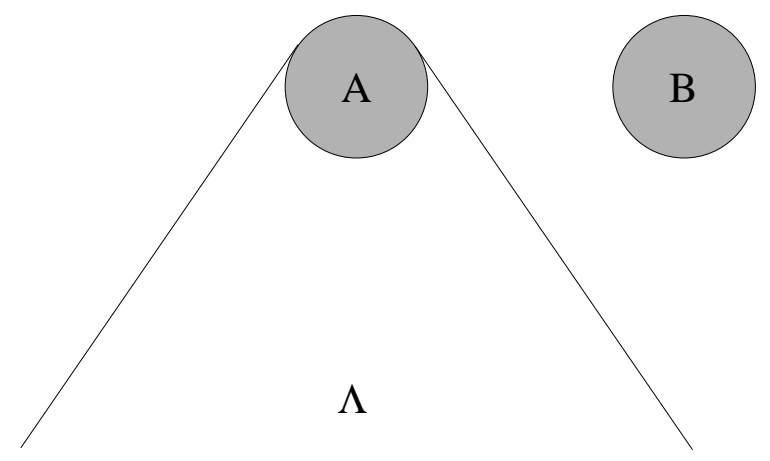

Figure 2: Local causality I.

should not be 'causes' of events in A, and vice versa. But this does not mean that the two sets of events should be uncorrelated, for they could have common causes in the overlap of their backward light cones. It is perfectly intelligible then that if $\Lambda$ in (6) does not contain a complete record of events in that overlap, it can be usefully supplemented by information from region B. So in general it is expected that

$$
p(A \mid \Lambda, B) \neq p(A \mid \Lambda)
$$

However, in the particular case that $\Lambda$ contains already a complete specification of beables in the overlap of the light cones, supplementary information from region B could reasonably be expected to be redundant."

And here comes the definition of a locally causal theory.

"Let $C$ denote a specification of all beables, of some theory, belonging to the overlap of the backward light cones of spacelike regions A and B. Let $a$ be a specification of some beables

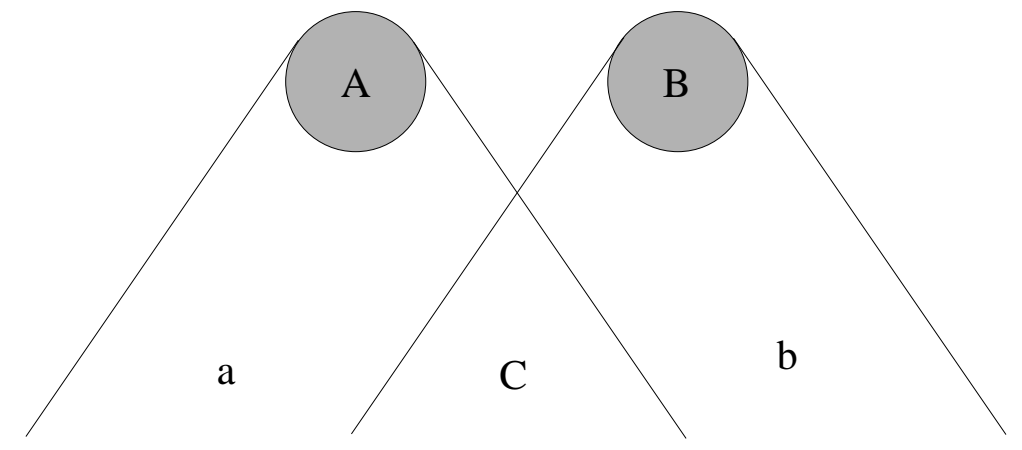

Figure 3: Local causality II.

\footnotetext{
${ }^{2}$ For the sake of uniformity throughout the paper, I slightly changed Bell's notation and figures.
} 
from the remainder of the backward light cone of $\mathrm{A}$, and $B$ of some beables in the region $\mathrm{B}$. (See Fig. 3.) Then in a locally causal theory

$$
p(A \mid a, C, B)=p(A \mid a, C)
$$

whenever both probabilities are given by the theory." (Bell, 1975/2004, p. 54)

Now, let us spell out Bell's characterization of local causality in our isotone net framework. To this end we need to 'translate' a number of terms Bell uses in his formulation into our language.

First, we need to translate Bell's language using random variables in (7) into a language using events. This is straightforward since events are special random variables, namely characteristic functions.

Second, we are to interpret the term 'beable'. 'Beable' is Bell's neologism and is contrasted to the term 'observable' used in quantum theory. "The beables of the theory are those entities in it which are, at least tentatively, to be taken seriously, as corresponding to something real" (Bell, 1990/2004, p. 234). Without the clarification of what the "beables" of a given theory really are, one cannot even formulate local theory since "there are things which do go faster than light. British sovereignty is the classical example. When the Queen dies in London (long may it be delayed) the Prince of Wales, lecturing on modern architecture in Australia, becomes instantaneously King" (Bell, 1990/2004, p. 236). In order to vitiate such 'violation' of local causality, the clarification of the "beables" of a theory is indispensable. (Cf. Norsen 2011.) What are the beables in the isotone net structure? Since these nets are classical and hence they represent objective physical events, any element of any local algebra will be regarded here as a beable.

Third, translating 'beable' simply as 'elements of an algebra' naturally brings with it the translation of the term 'complete specification of beables' as an 'atom of the algebra in question'. Here of course it is assumed that the local algebras of the net are atomic (which is typically not the case in AQFT). (For the translation of 'complete specification' into atomicity see (Henson, 2013a, p. 1015).)

Finally, an important point. Both in his wording and also in his figures Bell seems to take into account the whole causal past of the events in question. In the formulation of local causality he does not assume some kind of Markovian condition rendering superfluous the infinite tail of the past regions below a certain Cauchy surface. Other parts of Bell's text, however, speak for a more local interpretation of beable. ${ }^{3}$ Moreover, Bell's La nouvelle cuisine (Bell, 1990/2004), a posthumous paper on the same subject provides another definition of local causality where the screener-off regions are definitely finite. This definition is closer in spirit to the formalism of isotone nets since here only bounded regions are associated to local algebras. Therefore, we will here endorse this "finite" reading of local causality. (We will come back to this point in the Conclusion and discussion.)

With this 'translation manual' in hand, Bell's notion of local causality can be paraphrased as follows.

Definition 1. An isotone net $\mathfrak{N}$ associating bounded regions of the Minkowski spacetime to $\sigma$-subalgebras of $\Sigma$ is called locally causal, if for any classical probability measure $p$ on $\Sigma^{4}$, and for any two events $A_{m} \in \mathcal{A}\left(V_{A}\right)$ and $B_{n} \in \mathcal{A}\left(V_{B}\right)$ localized in the spacelike separated regions $V_{A}$ and $V_{B}$ and correlating in the probability measure $p$, the following holds.

Let $V_{a}, V_{b}$ and $V_{C}$ be three spacetime regions (see Fig. 4) such that

\footnotetext{
${ }^{3} \mathrm{Cf}$. "We will be particularly concerned with local beables, those which (unlike for example the total energy) can be assigned to some bounded [my italics] space-time region." (Bell, 1975/2004, p. 53)

${ }^{4} \mathrm{Or}$, in the more general AQFT case (which we do not need now): for any state $\phi$ on the quasilocal algebra $\mathcal{A}$. (Cf. Section 1 above.)
} 


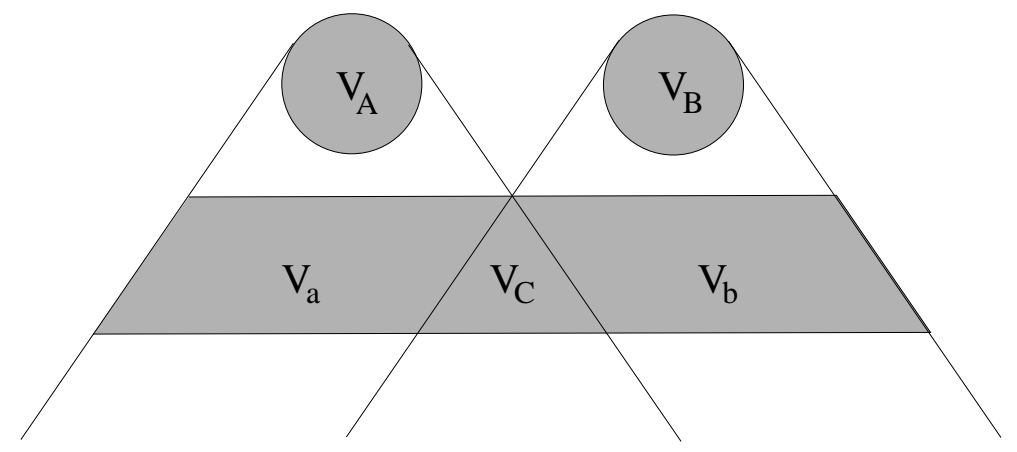

Figure 4: Local causality in isotone nets

$$
\begin{array}{r}
V_{a} \subset\left(I_{-}\left(V_{A}\right) \backslash I_{-}\left(V_{B}\right)\right) \\
V_{b} \subset\left(I_{-}\left(V_{B}\right) \backslash I_{-}\left(V_{A}\right)\right) \\
V_{C} \subset \mathcal{P}^{S}\left(V_{A}, V_{B}\right) \\
V_{C} \subset \mathcal{P}^{S}\left(V_{a}, V_{b}\right) \\
V_{A} \subset\left(V_{a} \cup V_{C}\right)^{\prime \prime} \\
V_{B} \subset\left(V_{C} \cup V_{b}\right)^{\prime \prime}
\end{array}
$$

Let $a_{m}, b_{n}$ and $C_{k}$ be any three atoms of the algebras $\mathcal{A}\left(V_{a}\right), \mathcal{A}\left(V_{b}\right)$ and $\mathcal{A}\left(V_{C}\right)$, respectively, associated to the appropriate regions. Then the following conditional probabilistic independences hold:

$$
\begin{aligned}
p\left(A_{m} \mid a_{m} \wedge C_{k} \wedge B_{n}\right) & =p\left(A_{m} \mid a_{m} \wedge C_{k}\right) \\
p\left(B_{n} \mid A_{m} \wedge C_{k} \wedge b_{n}\right) & =p\left(B_{n} \mid b_{n} \wedge C_{k}\right) \\
p\left(A_{m} \mid a_{m} \wedge C_{k} \wedge b_{n}\right) & =p\left(A_{m} \mid a_{m} \wedge C_{k}\right) \\
p\left(B_{n} \mid a_{m} \wedge C_{k} \wedge b_{n}\right) & =p\left(B_{n} \mid b_{n} \wedge C_{k}\right)
\end{aligned}
$$

Why four equations instead of Bell's single (7)? Observe that (15) is just the symmetric version of (14) where $A_{m}$ and $a_{m}$ are interchanged with $B_{n}$ and $b_{n}$. Equations (16)-(17), however, are slight extensions of Bell's formulation. Observe that $V_{A}$ is spacelike separated not only from $V_{B}$ but also from $V_{b}$; moreover, $V_{C}$ is in the strong past of $A$ and $B, \mathcal{P}^{S}\left(V_{A}, V_{b}\right)$. Therefore, conditioned on the complete specification of $V_{a} \cup V_{C}$, the same independence should hold between $A_{m}$ and $b_{n}$ as between $A_{m}$ and $B_{n}$. Thus (16) is the application of Bell's idea to algebras $\mathcal{A}\left(V_{A}\right)$ and $\mathcal{A}\left(V_{b}\right)$, and (17) to algebras $\mathcal{A}\left(V_{b}\right)$ and $\mathcal{A}\left(V_{A}\right)$. There are no more spacelike separated regions in Fig. 4 to which local causality could be applied.

How do the above considerations relate to the probabilistic characterization (2)-(5) of the common cause delineated in the previous Section?

First observe that (16)-(17) are equivalent to locality (3)-(4) and from (14)-(17) screening-off (2) follows directly. This proves that the probabilistic characterization of the common cause by the requirements of screening-off and locality can be 'derived' from Bell's notion of local causality imposed on an isotone net associating spacetime regions and local algebras.

There is, however, an important proviso. The third requirement in the definition of a common causal explanation, namely no-conspiracy (5) cannot be 'derived' from Bell's notion of local causality in a similar way. No-conspiracy is an independent assumption stating that the events $a_{m} \wedge b_{n}$ and $C_{k}$ are probabilistically independent. 
Let us come back for a moment to the definition of a locally causal net. In Definition 1 we required (14)-(17) and hence (2)-(4) to hold only for the atoms $a_{m}$ and $C_{k}$ of the algebras $\mathcal{A}\left(V_{a}\right)$ and $\mathcal{A}\left(V_{C}\right)$, respectively. Bell's original definition, however, seems to be more stringent; here (7) is required not only for the atoms of $\mathcal{A}\left(V_{a}\right)$ but for any element. This might suggest that our definition is weaker than that of Bell. This, however, is not the case. In Proposition 3 at the end of the paper we will show that in a locally causal net (2)-(4) hold not only for the atomic events $a_{m}, b_{n}$ and $C_{k}$, but (given some independence condition) also for any Boolean combination $a:=\vee_{m \in M^{\prime}} a_{m}, b:=\vee_{n \in N^{\prime}} a_{m}\left(M^{\prime} \subseteq M, N^{\prime} \subseteq N\right)$ of the measurement conditions. Note, however, that the common cause system $C_{k}$ cannot be 'aggregated' in this way: (2)-(4) will not necessarily hold for the Boolean combination $C:=\vee_{k \in K^{\prime}} C_{k}\left(K^{\prime} \subseteq K\right)$. This is why it is necessary to demand atomicity ("complete specification") in the strong past of the correlated events and sufficient to demand it outside it. We will come back to this point later.

An interesting question with respect to AQFT is the following. What is the relation between local primitive causality as standardly used in AQFT and our definition of local causality? The answer is given in the following proposition:

Proposition 1. A classical, atomic isotone net which satisfies local primitive causality $\left(\mathcal{A}(V)=\mathcal{A}\left(V^{\prime \prime}\right)\right.$ for any region $V$ ), automatically satisfies also local causality (14)-(17) for events in regions as shown in Fig. 4.

Proof. Consider first (14). Due to isotony and local primitive causality $\mathcal{A}\left(V_{A}\right) \subset \mathcal{A}\left(\left(V_{a} \cup V_{C}\right)^{\prime \prime}\right)=$ $\mathcal{A}\left(V_{a} \cup V_{C}\right)$ and hence for any atom $a_{m} \wedge C_{k}$ of $\mathcal{A}\left(V_{a} \cup V_{C}\right)$ : either (i) $A_{m} \wedge a_{m} \wedge C_{k}=0$ or (ii) $A_{m} \wedge a_{m} \wedge C_{k}=a_{m} \wedge C_{k}$. In case (i) both sides of (14) is zero, in case (ii) both sides of (14) is one. One obtains (15)-(17) in a similar fashion.

Intuitively, isotony and local primitive causality together ensure that the atoms of $\mathcal{A}\left(V_{a} \cup V_{C}\right)$ will also be atoms of $\mathcal{A}\left(V_{A}\right)$, hence screening off every correlation. For a more general proposition stating that in any atomic classical or quantum isotone net satisfying local primitive causality local causality also holds, see (Hofer-Szabó and Vecsernyés 2014a, Prop. 1) and (Hofer-Szabó and Vecsernyés 2014b, Sec. 3). For relating local causality (Stochastic Einstein Locality) to the axioms of AQFT (treated in the tradition of the so-called syntactical view of scientific theories), see (Rédei 1991) and (Muller and Butterfield 1994).

Reading Bell's formulation of local causality carefully, two ingredients of the definition stand out clearly. The one is that (i) the common cause system provides "a complete specification of beables", and (ii) it is located in the "overlap of the light cones". In our terminology, (i) $C_{k}$ is an atom of the appropriate algebra, (ii) it is located in the strong past of the correlated events. Bell explicitly stresses both points, and in all the subsequent papers of Van Fraassen (1982), Jarrett (1984), Shimony (1986) etc. trying to turn spacetime considerations into probabilistic independences these two requirements have been (explicitly or implicitly) made.

However, neither requirements are a priori concerning the idea of a common cause. One can easily make up common causes which are either non-atomic or not located in the strong past of the correlated events. How do these common causes relate to Bell's notion of local causality? In the following two Sections the relation between local causality and probabilistic characterization of the common cause will be studied first in the case of non-atomic common causes, then in case of weak common causes. In each Section toy models will be introduced first, then the formal results will be gathered.

\section{Non-atomic common causes}

Example 1. Consider the following toy model. There are five lighthouses on the ocean in a line at equal distances from one another. (See Fig. 5.) Let us count them from left to right. In the middle one, that is in lighthouse 3 the lighthouse keeper $\mathrm{C}$ has three lamps, $C^{\prime}, C^{\prime \prime}$ and $C^{\prime \prime \prime}$. He has the following strategy for turning the lamps on: either he turns on only the lamp $C^{\prime}$, or only lamp $C^{\prime \prime \prime}$, or all three lamps, or 

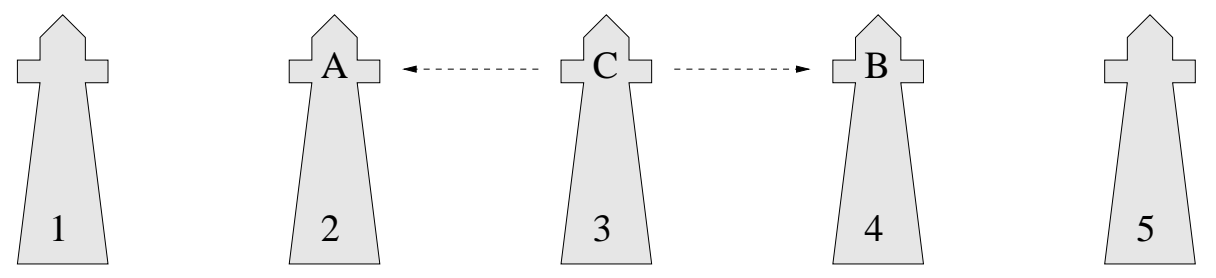

Figure 5: Lighthouses I.

none. He never turns on the lamps in any other combination. He chooses between these four options with equal probability (say, by tossing two coins). Let us denote that a given lamp is turned on and off by $C$ and $\bar{C}$, respectively. Using this notation the four possible state of the lamps are the following:

$$
\begin{aligned}
& C_{1} \equiv C^{\prime} \wedge \bar{C}^{\prime \prime} \wedge \bar{C}^{\prime \prime \prime} \\
& C_{2} \equiv \bar{C}^{\prime} \wedge \bar{C}^{\prime \prime} \wedge C^{\prime \prime \prime} \\
& C_{3} \equiv C^{\prime} \wedge C^{\prime \prime} \wedge C^{\prime \prime \prime} \\
& C_{4} \equiv \bar{C}^{\prime} \wedge \bar{C}^{\prime \prime} \wedge \bar{C}^{\prime \prime \prime}
\end{aligned}
$$

each with probability

$$
p\left(C_{k}\right)=\frac{1}{4}
$$

Now, in the left neighboring lighthouse, that is in lighthouse 2, there is another lighthouse keeper, A; and his role is simply to watch the light signals arriving from either the left or from the right, that is from either lighthouse 1 or lighthouse 3 . He does not know that lighthouse 1 is empty, therefore he spends equal time watching both neighboring lighthouses. Suppose furthermore that if he is watching to the left, he will miss the light signals coming from the right. This means that with probability $\frac{1}{2}$ he observes the signals coming from lighthouse 3 and with probability $\frac{1}{2}$ he will miss them. Denoting the event that the lighthouse keeper $\mathrm{A}$ is watching to the left and to the right by $a_{L}$ and $a_{R}$, respectively and denoting by $A$ the event that he observes a light signal (disregarding from which lamp it comes), one obtains the following conditional probabilities:

$$
p\left(A \mid a_{m} \wedge C_{k}\right)= \begin{cases}1 & \text { if } m=R, k=1,2,3 \\ 0 & \text { otherwise }\end{cases}
$$

In other words, the lighthouse keeper A observes the light signal only if he is watching right and there is a signal sent from C.

Suppose that the same thing happens also in lighthouse 4. The lighthouse keeper B is watching in both directions with equal probability, but since lighthouse 5 is empty, he misses the light signal coming from lighthouse 3 with probability $\frac{1}{2}$. Denoting again the events that the lighthouse keeper B is watching to the left and to the right by $b_{L}$ and $b_{R}$, respectively and denoting by $B$ the event that he observes a signal, one obtains the following conditional probabilities for B's observing a light signal:

$$
p\left(B \mid b_{n} \wedge C_{k}\right)= \begin{cases}1 & \text { if } n=L, k=1,2,3 \\ 0 & \text { otherwise. }\end{cases}
$$

This situation completely characterizes a probability space. The event algebra is generated by the following events:

$$
A, \bar{A}, B, \bar{B}, a_{m}, b_{n}, C_{k}
$$


with $m, n=L, R$ and $k=1,2,3,4$. The event algebra has 64 atoms, 16 of which have non-zero probability:

$$
\begin{aligned}
& p\left(A \wedge B \wedge a_{m} \wedge b_{n} \wedge C_{k}\right)=\frac{1}{16} \quad \text { if } m=R, n=L, k=1,2,3 \\
& p\left(A \wedge \bar{B} \wedge a_{m} \wedge b_{n} \wedge C_{k}\right)=\frac{1}{16} \quad \text { if } m, n=R, k=1,2,3 \\
& p\left(\bar{A} \wedge B \wedge a_{m} \wedge b_{n} \wedge C_{k}\right)=\frac{1}{16} \quad \text { if } m, n=L, k=1,2,3 \\
& p\left(\bar{A} \wedge \bar{B} \wedge a_{m} \wedge b_{n} \wedge C_{k}\right)=\frac{1}{16} \quad \text { if }\left\{\begin{array}{l}
m=L, n=R, k=1,2,3, \\
\text { or } k=4
\end{array}\right.
\end{aligned}
$$

and the remaining 48 are of probability zero. By means of the probability of the atoms one can easily calculate the probability of any events of the algebra.

Now, it is easy to see that there is a correlation between events $A$ and $B$ that is between the lighthouse keepers' observing a light signal, both in the non-conditional and conditional sense:

$$
\begin{aligned}
\frac{3}{16}=p(A \wedge B) & \neq p(A) p(B)=\frac{3}{8} \cdot \frac{3}{8} \\
\frac{3}{4}=p\left(A \wedge B \mid a_{m} \wedge b_{n}\right) & \neq p\left(A \mid a_{m}\right) p\left(B \mid b_{n}\right)=\frac{3}{4} \cdot \frac{3}{4} \quad \text { if } m=R, n=L
\end{aligned}
$$

As one expects, the correlation is due to C's signaling: $C_{k}$ is a local, (non-conspiratorial) joint common causal explanation of the correlation (26) in the sense of (2)-(5):

$$
\begin{aligned}
p\left(A \wedge B \mid a_{m} \wedge b_{n} \wedge C_{k}\right)=p\left(A \mid a_{m} \wedge b_{n} \wedge C_{k}\right) p\left(B \mid a_{m} \wedge b_{n} \wedge C_{k}\right) & = \begin{cases}1 & \text { if } m=R, n=L, k=1,2,3 \\
0 & \text { otherwise }\end{cases} \\
p\left(A \mid a_{m} \wedge b_{n} \wedge C_{k}\right)=p\left(A \mid a_{m} \wedge b_{n^{\prime}} \wedge C_{k}\right) & = \begin{cases}1 & \text { if } m=R, k=1,2,3 \\
0 & \text { otherwise }\end{cases} \\
p\left(B \mid a_{m} \wedge b_{n} \wedge C_{k}\right)=p\left(B_{n} \mid a_{m^{\prime}} \wedge b_{n} \wedge C_{k}\right) & = \begin{cases}1 & \text { if } n=L, k=1,2,3 \\
0 & \text { otherwise }\end{cases} \\
p\left(a_{m} \wedge b_{n} \wedge C_{k}\right)=p\left(a_{m} \wedge b_{n}\right) p\left(C_{k}\right) & =\frac{1}{4} \cdot \frac{1}{4}
\end{aligned}
$$

Example 2. Suppose we take a coarser clustering of the switching of the lamps, say $D_{1} \equiv C_{1} \vee C_{2} \vee C_{3}$ and $D_{2} \equiv C_{4}$. Physically, $D_{1}$ is the event that any light is on in lighthouse 3 , and $D_{2}$ is the event that no light is on. As one expects, for this coarser partition the common cause equations (2)-(5) will hold just as well as for the partition $\left\{C_{k}\right\}$ :

$$
\begin{aligned}
p\left(A \wedge B \mid a_{m} \wedge b_{n} \wedge D_{k}\right)=p\left(A \mid a_{m} \wedge b_{n} \wedge D_{k}\right) p\left(B \mid a_{m} \wedge b_{n} \wedge D_{k}\right) & = \begin{cases}1 & \text { if } m=R, n=L, k=1 \\
0 & \text { otherwise }\end{cases} \\
p\left(A \mid a_{m} \wedge b_{n} \wedge D_{k}\right)=p\left(A \mid a_{m} \wedge b_{n^{\prime}} \wedge D_{k}\right) & = \begin{cases}1 & \text { if } m=R, k=1 \\
0 & \text { otherwise }\end{cases} \\
p\left(B \mid a_{m} \wedge b_{n} \wedge D_{k}\right)=p\left(B_{n} \mid a_{m^{\prime}} \wedge b_{n} \wedge D_{k}\right) & = \begin{cases}1 & \text { if } n=L, k=1 \\
0 & \text { otherwise }\end{cases} \\
p\left(a_{m} \wedge b_{n} \wedge D_{k}\right)=p\left(a_{m} \wedge b_{n}\right) p\left(D_{k}\right) & = \begin{cases}\frac{1}{4} \cdot \frac{3}{4} & \text { if } n=L, k=1 \\
\frac{1}{4} \cdot \frac{1}{4} & \text { otherwise }\end{cases}
\end{aligned}
$$

Thus, $\left\{D_{k}\right\}$ is also a local, (non-conspiratorial) joint common causal explanation of the correlation (26). 
Example 3. Now, consider a coarser clustering of the switchings 'in the wrong way': $D_{1}^{\prime} \equiv C_{1} \vee C_{2} \vee C_{4}$ and $D_{2}^{\prime} \equiv C_{3}$ mixing together lights being on with lights being off. Contrary to the previous case, for this coarser partition the requirement of screening-off is violated. For example:

$$
\frac{2}{3}=p\left(A \wedge B \mid a_{R} \wedge b_{L} \wedge D_{1}^{\prime}\right) \quad \neq \quad p\left(A \mid a_{R} \wedge b_{L} \wedge D_{1}^{\prime}\right) p\left(B \mid a_{R} \wedge b_{L} \wedge D_{1}^{\prime}\right)=\frac{2}{3} \cdot \frac{2}{3}
$$

(Locality and no-conspiracy will hold even in this case.) Hence $\left\{D_{k}^{\prime}\right\}$ is not a local, (non-conspiratorial) joint common causal explanation of the correlation (26).

Now, let us consider the spacetime diagram of the above examples depicted in Fig. 6 . Let $\mathfrak{N}$ be a locally

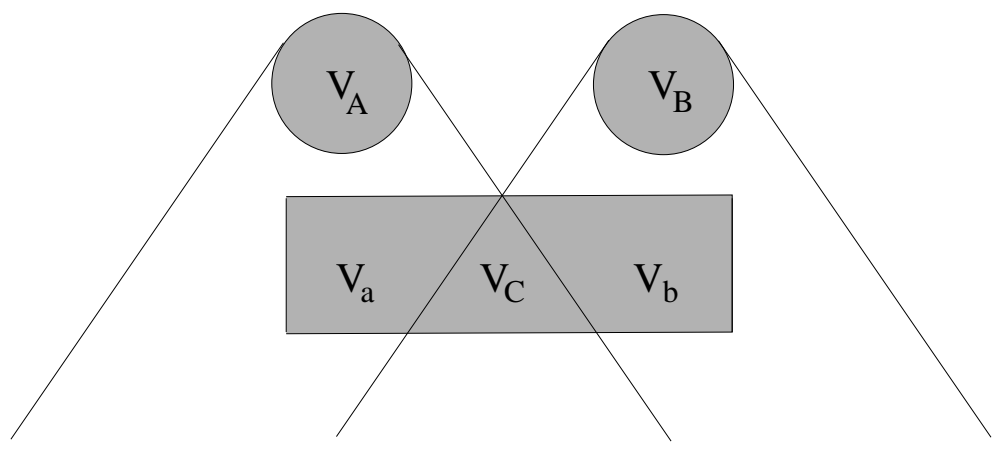

Figure 6: Spacetime diagram of Examples 1, 2 and 3.

causal net associating bounded spacetime regions to local algebras such that $A \in \mathcal{A}\left(V_{A}\right), B \in \mathcal{A}\left(V_{B}\right)$, $a_{m} \in \mathcal{A}\left(V_{a}\right), b_{n} \in \mathcal{A}\left(V_{b}\right)$ and $C_{k}, D_{k}, D_{k}^{\prime} \in \mathcal{A}\left(V_{C}\right)$ for all $m, n$ and $k$. As shown in Section 2, local causality of the net implies that the set $\left\{C_{k}\right\}$-being an atomic partition localized in the strong past $\mathcal{P}^{S}\left(V_{A}, V_{B}\right)$-satisfies (2)-(4), hence providing a local, joint common causal explanation of the correlation (26). (No-conspiracy (5), as already stressed in Section 2, is not a consequence of local causality but is assumed in the toy model.) Thus, $\left\{C_{k}\right\}$ is an atomic, strong, local, non-conspiratorial joint common cause system.

What about non-atomic partitions localized in the strong past? Again, both $\left\{D_{k}\right\}$ and $\left\{D_{k}^{\prime}\right\}$ are localized in $\mathcal{P}^{S}\left(V_{A}, V_{B}\right)$, but whereas $\left\{D_{k}\right\}$ is a common cause system of the correlation $(26)$, $\left\{D_{k}^{\prime}\right\}$ is not. Thus, local causality is completely silent about whether a coarse-grained partition of a local algebra in the strong past is a common cause system of the correlated events or not. This 'non-aggregable' character of the atomic common cause relies heavily on the fact that it is localized in the strong past-as will be seen in Proposition 3 in the next Section when contrasted with the opposite character of weak common causes. Moreover, the satisfaction of equations (2)-(5) for a given partition also does not ensure that finer-grained partitions will also do so (this is Simpson's paradox; see e.g. (Uffink 1999)). In this sense the existence of a common cause system characterized by the probabilistic constraints (2)-(5) for a given correlation is a weaker requirement than the accommodation of the same correlation in a locally causal theory. There are many more local, non-conspiratorial joint common cause systems than the atomic ones required by locally causal theories.

Obviously, from the perspective of the EPR-Bell scenario this difference is not of central importance, since the violation of the Bell inequalities derived from (2)-(5) also excludes atomic common cause systems and hence the possibility of a locally causal theory. But focusing simply on the logical relation between Bell's local causality and the probabilistic equations (2)-(5), it is fair to say that local causality 'justifies' only one of the multiple common causal explanations, namely the atomic one. The coarse-grained common cause system $\left\{D_{k}\right\}$, however, is an entirely salient physical explanation of the the correlation ("Observers see light signals only if some lamps are switched on"), even if the existence of such a common causal 
explanation is not a consequence of the accommodation of the physical scenario into a locally causal theory.

Now we turn to the role of the other ingredient in Bell's formulation, namely the localization of the common cause in the strong past.

\section{$5 \quad$ Weak common causes}

Example 4. Now, let us modify the population of the lighthouses. Let A and B remain in their places, that is in lighthouse 2 and 4, respectively: but suppose that lighthouses 1,3 and 5 are inhabited by three lighthouse keepers $C^{\prime}, C^{\prime \prime}$ and $C^{\prime \prime \prime}$, respectively, each having the corresponding one of the three lamps introduced in the previous Section. (See Fig. 7.) That is suppose that now lighthouse keeper $C^{\prime}$

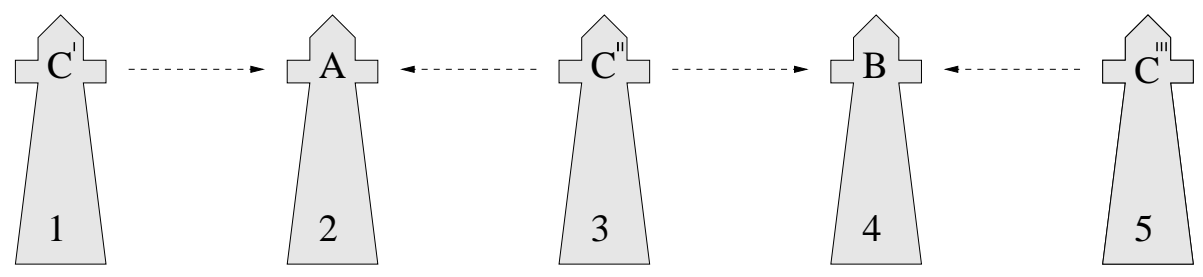

Figure 7: Lighthouses II.

in lighthouse 1 operates lamp $C^{\prime}$, lighthouse keeper $C^{\prime \prime}$ in lighthouse 3 operates lamp $C^{\prime \prime}$ and lighthouse keeper $C^{\prime \prime \prime}$ in lighthouse 5 operates lamp $C^{\prime \prime \prime}$. Suppose furthermore that the ons and offs of the different lamps follow just the same statistics as defined in (18)-(22), that is $p\left(C_{k}\right)=\frac{1}{4}$ for every $k=1,2,3,4$ (only lamp $C^{\prime}$ is on, only lamp $C^{\prime \prime \prime}$, all three lamps are on, none is on).

Now, the role of lighthouse keepers A and B is just as in Section 4: to watch the light signals arriving at lighthouse 2 and 4 , respectively. But now both can obtain a signal from both directions. Suppose that both A and B can only see the light signal sent from a neighboring lighthouse. That is, $A$ cannot see the signal sent from $C^{\prime \prime \prime}$ (say, because it is too far or the lighthouses hide each other); and $B$ cannot see the signal sent from $C^{\prime}$. Now, again the event algebra has 16 atoms with non-zero probability:

$$
\begin{array}{ll}
p\left(A \wedge B \wedge a_{m} \wedge b_{n} \wedge C_{k}\right)=\frac{1}{16} & \text { if } k=3 \\
p\left(A \wedge \bar{B} \wedge a_{m} \wedge b_{n} \wedge C_{k}\right)=\frac{1}{16} & \text { if } m=L, k=1 \\
p\left(\bar{A} \wedge B \wedge a_{m} \wedge b_{n} \wedge C_{k}\right)=\frac{1}{16} & \text { if } n=R, k=2 \\
p\left(\bar{A} \wedge \bar{B} \wedge a_{m} \wedge b_{n} \wedge C_{k}\right)=\frac{1}{16} & \text { if }\left\{\begin{array}{l}
m=R, k=1, \\
\text { or } n=L, k=2, \\
\text { or } k=4
\end{array}\right.
\end{array}
$$

and there is a conditional and non-conditional correlation between event $A$ and $B$, the detections of light signals in lighthouse 2 and 4 , respectively, both in the non-conditional and conditional sense:

$$
\begin{gathered}
\frac{1}{4}=p(A \wedge B) \neq p(A) p(B)=\frac{3}{8} \cdot \frac{3}{8} \\
\frac{1}{4}=p\left(A \wedge B \mid a_{m} \wedge b_{n}\right) \neq p\left(A \mid a_{m}\right) p\left(B \mid b_{n}\right)= \begin{cases}\frac{1}{4} \cdot \frac{1}{4} & \text { if } m=R, n=L, \\
\frac{1}{4} \cdot \frac{1}{2} & \text { if } m, n=R, \\
\frac{1}{2} \cdot \frac{1}{4} & \text { if } m, n=L .\end{cases}
\end{gathered}
$$


As one expects, $\left\{C_{k}\right\}$ is a local, (non-conspiratorial) joint common causal explanation of the correlation:

$$
\begin{aligned}
p\left(A \wedge B \mid a_{m} \wedge b_{n} \wedge C_{k}\right)=p\left(A \mid a_{m} \wedge b_{n} \wedge C_{k}\right) p\left(B \mid a_{m} \wedge b_{n} \wedge C_{k}\right) & = \begin{cases}1 & \text { if } m=R, n=L, k=3 \\
0 & \text { otherwise }\end{cases} \\
p\left(A \mid a_{m} \wedge b_{n} \wedge C_{k}\right)=p\left(A \mid a_{m} \wedge b_{n^{\prime}} \wedge C_{k}\right) & = \begin{cases}1 & \text { if } m=L, k=1 \\
1 & \text { if } k=3 \\
0 & \text { otherwise }\end{cases} \\
p\left(B \mid a_{m} \wedge b_{n} \wedge C_{k}\right)=p\left(B_{n} \mid a_{m^{\prime}} \wedge b_{n} \wedge C_{k}\right) & = \begin{cases}1 & \text { if } m=R, k=2 \\
1 & \text { if } k=3 \\
0 & \text { otherwise }\end{cases} \\
p\left(a_{m} \wedge b_{n} \wedge C_{k}\right)=p\left(a_{m} \wedge b_{n}\right) p\left(C_{k}\right) & =\frac{1}{4} \cdot \frac{1}{4}
\end{aligned}
$$

Now, consider again the spacetime diagram of Example 4 depicted in Fig. 8. Here $\left\{C_{k}\right\}$ is localized not

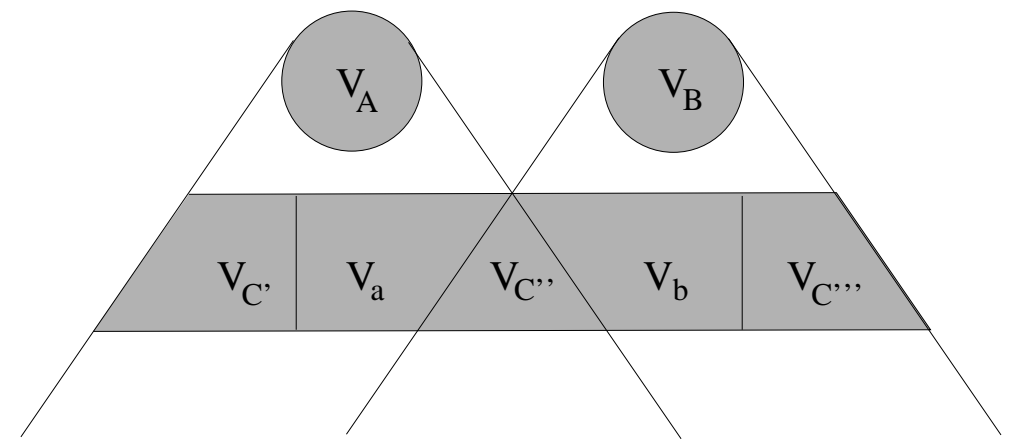

Figure 8: Spacetime diagram of Example 4.

in the strong past but in the weak past of the correlated events. How do these weak common causes relate to Bell's local causality? This question is answered in the following

Proposition 2. Let $\mathfrak{N}$ be again a locally causal net associating bounded spacetime regions to local algebras and let $A \in \mathcal{A}\left(V_{A}\right), B \in \mathcal{A}\left(V_{B}\right), a_{m} \in \mathcal{A}\left(V_{a}\right), b_{n} \in \mathcal{A}\left(V_{b}\right), C_{i}^{\prime} \in \mathcal{A}\left(V_{C^{\prime}}\right), C_{j}^{\prime \prime} \in \mathcal{A}\left(V_{C^{\prime \prime}}\right)$ and $C_{l}^{\prime \prime \prime} \in \mathcal{A}\left(V_{C^{\prime \prime \prime}}\right)$ for all $m, n, i, j, l$ be atoms of the appropriate algebras with the regions as shown in Fig. 8. (In Example $4 C_{1}^{\prime} \equiv C^{\prime}, C_{2}^{\prime} \equiv \bar{C}^{\prime}$ and similarly for $C_{j}^{\prime \prime}$ and $C_{l}^{\prime \prime \prime}$.) Then

$$
\left\{C_{i j l}\right\} \equiv\left\{C_{i}^{\prime} \wedge C_{j}^{\prime \prime} \wedge C_{l}^{\prime \prime \prime}\right\}
$$

is a weak, local, joint common cause of the conditional correlations

$$
p\left(A \wedge B \mid a_{m} \wedge b_{n}\right) \neq p\left(A \mid a_{m}\right) p\left(B \mid b_{n}\right)
$$

in the sense that the following equations hold:

$$
\begin{aligned}
p\left(A \wedge B_{n} \mid a_{m} \wedge b_{n} \wedge C_{i j l}\right) & =p\left(A \mid a_{m} \wedge b_{n} \wedge C_{i j l}\right) p\left(B \mid a_{m} \wedge b_{n} \wedge C_{i j l}\right) \\
p\left(A \mid a_{m} \wedge b_{n} \wedge C_{i j l}\right) & =p\left(A \mid a_{m} \wedge b_{n^{\prime}} \wedge C_{i j l}\right) \\
p\left(B \mid a_{m} \wedge b_{n} \wedge C_{i j l}\right) & =p\left(B \mid a_{m^{\prime}} \wedge b_{n} \wedge C_{i j l}\right)
\end{aligned}
$$


Proof. The proof is straightforward. Local causality of the net implies that for the atoms $a_{i m}^{\prime} \equiv$ $C_{i}^{\prime} \wedge a_{m} \in \mathcal{A}\left(V_{C^{\prime}} \cup V_{a}\right), b_{n l}^{\prime} \equiv b_{n} \wedge C_{l}^{\prime \prime \prime} \in \mathcal{A}\left(V_{b} \cup V_{C^{\prime \prime \prime}}\right)$ and $C_{j}^{\prime \prime} \in \mathcal{A}\left(V_{C^{\prime \prime}}\right)$ the following equations hold (being analogous to local causality (14)-(17)):

$$
\begin{aligned}
p\left(A \wedge B_{n} \mid a_{i m}^{\prime} \wedge b_{n l}^{\prime} \wedge C_{j}^{\prime \prime}\right) & =p\left(A \mid a_{i m}^{\prime} \wedge b_{n l}^{\prime} \wedge C_{j}^{\prime \prime}\right) p\left(B \mid a_{i m}^{\prime} \wedge b_{n l}^{\prime} \wedge C_{j}^{\prime \prime}\right) \\
p\left(A \mid a_{i m}^{\prime} \wedge b_{n l}^{\prime} \wedge C_{j}^{\prime \prime}\right) & =p\left(A \mid a_{i m}^{\prime} \wedge b_{n^{\prime} l^{\prime}}^{\prime} \wedge C_{j}^{\prime \prime}\right) \\
p\left(B \mid a_{i m}^{\prime} \wedge b_{n l}^{\prime} \wedge C_{j}^{\prime \prime}\right) & =p\left(B \mid a_{i^{\prime} m^{\prime}}^{\prime} \wedge b_{n l}^{\prime} \wedge C_{j}^{\prime \prime}\right)
\end{aligned}
$$

In other words, $\left\{C_{j}^{\prime \prime}\right\}$ is a strong, local, joint common cause of the conditional correlations

$$
p\left(A \wedge B \mid a_{i m}^{\prime} \wedge b_{n l}^{\prime}\right) \neq p\left(A \mid a_{i m}^{\prime}\right) p\left(B \mid b_{n l}^{\prime}\right)
$$

with the new conditions $a_{i m}^{\prime}$ and $b_{n l}^{\prime}$. (Again, no-conspiracy

$$
p\left(a_{i m}^{\prime} \wedge b_{n l}^{\prime} \wedge C_{j}^{\prime \prime}\right)=p\left(a_{i m}^{\prime} \wedge b_{n l}^{\prime}\right) p\left(C_{j}^{\prime \prime}\right)
$$

does not follow from local causality of the net.) But (33)-(35) are just equivalent to (30)-(32) proving that $\left\{C_{i j l}\right\}$ is a weak, local, joint common cause of the conditional correlations (29).

As we saw before, the correlated events $A \in \mathcal{A}\left(V_{A}\right), B \in \mathcal{A}\left(V_{B}\right)$ in a locally causal net always have an atomic, strong common cause system $C_{j}^{\prime \prime} \in \mathcal{A}\left(V_{C^{\prime \prime}}\right)$. Now, Proposition 2 states that this strong common cause system can always be spatially extended into a weak common cause system by simply adding some elements $C_{i}^{\prime}$ and $C_{l}^{\prime \prime \prime}$ from the spacelike separated regions $V_{C^{\prime}}$ and $V_{C^{\prime \prime \prime}}$, respectively. These extra terms will not spoil the screening-off: they can be freely added to the strong common cause. Moreover, as will turn out from Proposition 3, these extra terms need not be atomic either: any Boolean combination $C^{\prime}=\vee_{i} C_{i}^{\prime}$ and $C^{\prime \prime \prime}=\vee_{l} C_{l}^{\prime \prime \prime}$ can also be added without violating the probabilistic constraints (2)-(4). Thus, local causality does not determine the localization of the common cause, it is compatible both with strong and weak common causes.

But what is the exact relation between the weak and the strong common cause systems arising from the local causality of a given net?

In Example 4 one might find it peculiar that even though the common cause $\left\{C_{i j l}\right\}$ was nonconspiratorial (it was probabilistically independent of $a_{m}$ and $b_{n}$ ), still there was a 'conspiracy' within the common cause: $C_{i}^{\prime}, C_{j}^{\prime \prime}$ and $C_{l}^{\prime \prime \prime}$ were not probabilistically independent. For example it never happened that only lamp $C^{\prime \prime}$ was switched on. This fact does not raise any problem until one asks whether the common cause is localized at one place: for example, as in Example 1, where all the three lamps were localized in lighthouse 3. But in Example 4 the common cause was scattered around in three different locations. It was located in three different lighthouses. The problem with such a common cause that it may well question our whole project to provide a common causal explanation for a correlation. If the explanans itself has a built-in correlation, then what is the point in using it for explaining correlations? Can we not come up with a common causal model in which $C_{i}^{\prime}, C_{j}^{\prime \prime}$ and $C_{l}^{\prime \prime \prime}$ are spacelike separated but still independent, say, regulated by three independent coin tossings in lighthouse 1,3 and 5 , respectively. Can one obtain a weak common cause for a given correlation without a built-in correlation? In the next proposition we will answer this question in the negative.

Let $\left\{C_{i j l}\right\} \equiv\left\{C_{i}^{\prime} \wedge C_{j}^{\prime \prime} \wedge C_{l}^{\prime \prime \prime}\right\}$ be a weak common cause of a given correlation. (Here $\left\{C_{i}^{\prime}\right\},\left\{C_{j}^{\prime \prime}\right\}$ and $\left\{C_{l}^{\prime \prime \prime}\right\}$ are general partitions of $\mathcal{A}\left(V_{C^{\prime}}\right), \mathcal{A}\left(V_{C^{\prime \prime}}\right)$ and $\mathcal{A}\left(V_{C^{\prime \prime \prime}}\right)$, respectively, and not those special ones specified in the above Examples.) Let us call $\left\{C_{i j l}\right\}$ a genuine weak common cause, iff $\left\{C_{j}^{\prime \prime}\right\}$-the 'middle part' of $\left\{C_{i j l}\right\}$-is not a strong common cause. In what follows we will show that the above mentioned 'built-in correlation' is a necessary condition to explain a correlation by a genuine weak common cause. In other words, we will show that if $\left\{C_{i j l}\right\} \equiv\left\{C_{i}^{\prime} \wedge C_{j}^{\prime \prime} \wedge C_{l}^{\prime \prime \prime}\right\}$ is a common cause of the correlation (29) and $C_{i}^{\prime}, C_{j}^{\prime \prime}$ and $C_{l}^{\prime \prime \prime}$ are probabilistically independent, then also $\left\{C_{j}^{\prime \prime}\right\}$ will be a common cause of the correlation. 
Proposition 3. Suppose that $\left\{C_{i}^{\prime} \wedge C_{j}^{\prime \prime} \wedge C_{l}^{\prime \prime \prime}\right\}$ is a common cause of the correlation between $A_{m}$ and $B_{n}$ in the sense that the following equations hold:

$$
\begin{aligned}
p\left(A_{m} \wedge B_{n} \mid a_{m} \wedge b_{n} \wedge C_{i}^{\prime} \wedge C_{j}^{\prime \prime} \wedge C_{l}^{\prime \prime \prime}\right) & =p\left(A_{m} \mid a_{m} \wedge b_{n} \wedge C_{i}^{\prime} \wedge C_{j}^{\prime \prime} \wedge C_{l}^{\prime \prime \prime}\right) p\left(B_{n} \mid a_{m} \wedge b_{n} \wedge C_{i}^{\prime} \wedge C_{j}^{\prime \prime} \wedge\right. \\
p\left(A_{m} \mid a_{m} \wedge b_{n} \wedge C_{i}^{\prime} \wedge C_{j}^{\prime \prime} \wedge C_{l}^{\prime \prime \prime}\right) & =p\left(A_{m} \mid a_{m} \wedge b_{n^{\prime}} \wedge C_{i}^{\prime} \wedge C_{j}^{\prime \prime} \wedge C_{l}^{\prime \prime \prime}\right) \\
p\left(B_{n} \mid a_{m} \wedge b_{n} \wedge C_{i}^{\prime} \wedge C_{j}^{\prime \prime} \wedge C_{l}^{\prime \prime \prime}\right) & =p\left(B_{n} \mid a_{m^{\prime}} \wedge b_{n} \wedge C_{i}^{\prime} \wedge C_{j}^{\prime \prime} \wedge C_{l}^{\prime \prime \prime}\right) \\
p\left(a_{m} \wedge b_{n} \wedge C_{i}^{\prime} \wedge C_{j}^{\prime \prime} \wedge C_{l}^{\prime \prime \prime}\right) & =p\left(a_{m} \wedge b_{n}\right) p\left(C_{i}^{\prime} \wedge C_{j}^{\prime \prime} \wedge C_{l}^{\prime \prime \prime}\right)
\end{aligned}
$$

and suppose that $C_{i}^{\prime}, C_{j}^{\prime \prime}$ and $C_{l}^{\prime \prime \prime}$ are independent, that is

$$
p\left(C_{i}^{\prime} \wedge C_{j}^{\prime \prime} \wedge C_{l}^{\prime \prime \prime}\right)=p\left(C_{i}^{\prime}\right) p\left(C_{j}^{\prime \prime}\right) p\left(C_{l}^{\prime \prime \prime}\right)
$$

then $\left\{C_{j}^{\prime \prime}\right\}$ is also a common cause of the correlation:

$$
\begin{aligned}
p\left(A_{m} \wedge B_{n} \mid a_{m} \wedge C_{j}^{\prime \prime}\right) & =p\left(A_{m} \mid a_{m} \wedge b_{n} \wedge C_{j}^{\prime \prime}\right) p\left(B_{n} \mid a_{m} \wedge b_{n} \wedge C_{j}^{\prime \prime}\right) \\
p\left(A_{m} \mid a_{m} \wedge b_{n} \wedge C_{j}^{\prime \prime}\right) & =p\left(A_{m} \mid a_{m} \wedge b_{n^{\prime}} \wedge C_{j}^{\prime \prime}\right) \\
p\left(B_{n} \mid a_{m} \wedge b_{n} \wedge C_{j}^{\prime \prime}\right) & =p\left(B_{n} \mid a_{m^{\prime}} \wedge b_{n} \wedge C_{j}^{\prime \prime}\right) \\
p\left(a_{m} \wedge b_{n} \wedge C_{j}^{\prime \prime}\right) & =p\left(a_{m} \wedge b_{n}\right) p\left(C_{j}^{\prime \prime}\right)
\end{aligned}
$$

For the proof see Appendix B. Since in Example $4\left\{C_{i j l}\right\} \equiv\left\{C_{i}^{\prime} \wedge C_{j}^{\prime \prime} \wedge C_{l}^{\prime \prime \prime}\right\}$ was localized in the weak past and $\left\{C_{j}^{\prime \prime}\right\}$ was localized in the strong past, we can interpret Proposition 3 as follows: a weak common cause without a 'built-in correlation' is always 'parasitic' on a strong common cause in the sense that there is no other way to provide a genuine weak common cause for a given correlation than to make the spacelike separated parts of the common cause probabilistically dependent. In brief, there is no genuine weak common cause without 'built-in correlation'.

Proposition 3 nicely explains why we are compelled to use strong common causes in classical common causal explanations. If we want to avoid explaining correlations in terms of other correlations, we cannot apply genuine weak common causes. So instead of appealing to non-genuine ('parasitic') weak common causes, it is more informative to use simply strong common causes.

The type of the common cause, however, is not always a matter of what we might want. As was mentioned in the Introduction, the common causes that naturally arise in AQFT are weak and not strong common causes. Why is that? The mathematical answer, namely that only (the possibility of) weak common causes follows from the axioms of the theory (see (Rédei 1997) and also (Hofer-Szabó and Vecsernyés 2012a, b)), is not very intuitive. In search of a more intuitive explanation, we conclude this paper with a highly speculative question:

Question: Is the fact that common causes in AQFT are weak common causes somehow related to or a consequence of the following two facts? (If these latter are facts at all.)

1. In AQFT quantum states establishing a superluminal correlation between two spacelike separated events also establish (or 'typically' establish) a 'built-in correlation' between the spacelike separated parts of the weak common causes of this correlation.

2. An analogue of Proposition 3 holds in AQFT: stating that, roughly speaking, a 'built-in correlation' is a necessary condition to explain a correlation by a genuine weak common cause.

Were these two facts to hold, one could understand why weak common causes in AQFT are genuine common causes, that is why they do not reduce to strong common causes. (For more on this see (HoferSzabó and Vecsernyés 2014a, b).) 


\section{Conclusion and discussion}

In this paper, we gave a framework connecting stochastic events and spacetime regions, the isotone net framework of AQFT (Point 1) such that, on a certain specification and localization of the events in question (Point 2), local causality, defined in this framework in an appropriate way, implies (up to noconspiracy) the standard probabilistic characterization of the common causal explanation (Point 3). The subtle roles of the choice of specification (atomic vs. non-atomic) and localization (strong vs. weak) were analyzed with respect to the relations of the spatiotemporal and probabilistic characterizations of the common cause. Specifically, it was shown that (i) the existence of non-atomic probabilistic common causes does not follow from the accommodation of the correlations in question into a locally causal net; (ii) the probabilistic characterization of the common cause is also compatible with weak common causes; and (iii) genuine weak common causes can be provided for a given correlation only at the cost of introducing a 'built-in correlation' between the spacelike separated parts of the common cause. We also asked whether this latter fact can help us understand how weak common causes arise naturally in AQFT.

Finally, we would like to briefly comment on an ongoing debate between Henson, Rédei and San Pedro on "comparing-distinguishing-confounding causality principles" (Henson, 2005; Rédei and San Pedro, 2012; Henson, 2013a). The debate is about the status of a proposition proved in Henson (2005) claiming that the Strong and Weak Common Cause Principles are equivalent. Here Strong/Weak Common Cause Principles say that any atom of the algebra pertaining to the strong/weak past of a pair of correlated events is a screener-off. The use of atoms (there called "full specifications") in the Common Cause Principles is inspired-just as in this paper-by Bell's work (see also Norsen, 2011), and further motivated as a means to evade Simpson paradoxes (see also Uffink, 1999). The first point to make is that since Henson's framework connecting spacetime regions and probability spaces is not the isotone net formalism used in this paper, and his Common Cause Principles are not the non-conspiratorial, local, joint common causal explanation (2)-(5) (used to explain conditional correlations!), it is not easy to see how Henson's result exactly relates to ours. In the isotone net formalism only bounded regions are associated to local algebras, whereas Henson's "least domains of decidability" formalism is not restricted to such regions. Rédei and San Pedro (2012) challenge Henson's result on the basis of its incompatibility with some propositions in AQFT (Rédei and Summers, 2002, Proposition 3). They claim that Henson's proof crucially depends on the regions being allowed to be infinite; and they question the validity of a similar proof for finite regions. ${ }^{5}$ For finite regions, such as the regions in our approach, Henson acknowledges that his proof "cannot be modified so that" the two Common Cause Principles are equivalent; "at least not assuming that there are no correlations between events on spacelike sections of initial hypersurface" (Henson, 2005, 532). In the light of our results and discussion above, we would like to interpret: (i) the first part of this quote as claiming that (provided the two formalisms are equivalent) there is no contradiction between Henson's proof and our sharp distinction between weak and strong common causes; and (ii) the second half of the quote as stating something parallel to Proposition 3. Nonetheless, it would be highly desirable to investigate the relation between the two approaches more thoroughly.

Acknowledgements. I wish to thank Jeremy Butterfield, the Reading Group of Fellows in the Center for Philosophy of Science at the University of Pittsburgh, and the Castle Hill Reading Group in Philosophy of Science in the Institute of Philosophy at the Hungarian Academy of Sciences for their helpful comments. This work has been supported by the Hungarian Scientific Research Fund, OTKA K-100715 and by the Fulbright Research Grant while G. H-Sz. was a Visiting Fellow in the Center for Philosophy of Science at the University of Pittsburgh.

\footnotetext{
${ }^{5}$ Their characterization of "finite", however, is defective, since the region they want to have as infinite turns out to be finite; which fact is revealed in Henson's (2013a) reply. Here is a better characterization: $V$ is finite iff $\left(I_{-}\left(V^{\prime \prime}\right) \backslash\left(V^{\prime \prime}\right)\right)^{\prime \prime} \supseteq$ $V^{\prime \prime}$.
} 


\section{Appendix A}

Here we will show that if a set of correlations $\left\{\left(A_{m}, B_{n}\right) \mid m, n=1,2\right\}$ has a local, non-conspiratorial joint common causal explanation in the sense of (2)-(5), then the following Clauser-Horne inequalities have to hold for any $m, m^{\prime}, n, n^{\prime}=1,2 ; m \neq m^{\prime}, n \neq n^{\prime}$ :

$$
\begin{array}{r}
-1 \leqslant p\left(A_{m} \wedge B_{n} \mid a_{m} \wedge b_{n}\right)+p\left(A_{m} \wedge B_{n^{\prime}} \mid a_{m} \wedge b_{n^{\prime}}\right)+p\left(A_{m^{\prime}} \wedge B_{n} \mid a_{m^{\prime}} \wedge b_{n}\right) \\
-p\left(A_{m^{\prime}} \wedge B_{n^{\prime}} \mid a_{m^{\prime}} \wedge b_{n^{\prime}}\right)-p\left(A_{m} \mid a_{m} \wedge b_{n}\right)-p\left(B_{n} \mid a_{m} \wedge b_{n}\right) \leqslant 0
\end{array}
$$

The derivation of (47) from (2)-(5) is simple. It is an elementary fact of arithmetic that for any $\alpha, \alpha^{6}, \beta, \beta^{6} \in[0,1]$ the number

$$
\alpha \beta+\alpha \beta^{\natural}+\alpha^{\natural} \beta-\alpha^{\natural} \beta^{\natural}-\alpha-\beta
$$

lies in the interval $[-1,0]$. Now let $\alpha, \alpha^{6}, \beta, \beta^{6}$ be the following conditional probabilities:

$$
\begin{aligned}
\alpha & \equiv p\left(A_{m} \mid a_{m} \wedge b_{n} \wedge C_{k}\right) \\
\alpha^{6} & \equiv p\left(A_{m^{\prime}} \mid a_{m^{\prime}} \wedge b_{n^{\prime}} \wedge C_{k}\right) \\
\beta & \equiv p\left(B_{n} \mid a_{m} \wedge b_{n} \wedge C_{k}\right) \\
\beta^{6} & \equiv p\left(B_{n^{\prime}} \mid a_{m^{\prime}} \wedge b_{n^{\prime}} \wedge C_{k}\right)
\end{aligned}
$$

Plugging (49)-(52) into (48) and using locality (3)-(4) one obtains

$$
\begin{array}{r}
-1 \leqslant p\left(A_{m} \mid a_{m} \wedge b_{n} \wedge C_{k}\right) p\left(B_{n} \mid a_{m} \wedge b_{n} \wedge C_{k}\right)+p\left(A_{m} \mid a_{m^{\prime}} \wedge b_{n} \wedge C_{k}\right) p\left(B_{n^{\prime}} \mid a_{m^{\prime}} \wedge b_{n} \wedge C_{k}\right) \\
+p\left(A_{m^{\prime}} \mid a_{m^{\prime}} \wedge b_{n} \wedge C_{k}\right) p\left(B_{n} \mid a_{m^{\prime}} \wedge b_{n} \wedge C_{k}\right)-p\left(A_{m^{\prime}} \mid a_{m^{\prime}} \wedge b_{n^{\prime}} \wedge C_{k}\right) p\left(B_{n^{\prime}} \mid a_{m^{\prime}} \wedge b_{n^{\prime}} \wedge C_{k}\right) \\
-p\left(A_{m} \mid a_{m} \wedge b_{n} \wedge C_{k}\right)-p\left(B_{n} \mid a_{m} \wedge b_{n} \wedge C_{k}\right) \leqslant 0
\end{array}
$$

Using screening-off (2) one obtains

$$
\begin{array}{r}
-1 \leqslant p\left(A_{m} \wedge B_{n} \mid a_{m} \wedge b_{n} \wedge C_{k}\right)+p\left(A_{m} \wedge B_{n^{\prime}} \mid a_{m^{\prime}} \wedge b_{n} \wedge C_{k}\right) \\
+p\left(A_{m^{\prime}} \wedge B_{n} \mid a_{m^{\prime}} \wedge b_{n} \wedge C_{k}\right)-p\left(A_{m^{\prime}} \wedge B_{n^{\prime}} \mid a_{m^{\prime}} \wedge b_{n^{\prime}} \wedge C_{k}\right) \\
-p\left(A_{m} \mid a_{m} \wedge b_{n} \wedge C_{k}\right)-p\left(B_{n} \mid a_{m} \wedge b_{n} \wedge C_{k}\right) \leqslant 0
\end{array}
$$

Finally, multiplying the above inequality by $p\left(C_{k}\right)$, then summing up for the indices $k$ and using noconspiracy (5) one arrives at (47).

\section{Appendix B}

Here we prove Proposition 2. Suppose that $\left\{C_{i}^{\prime} \wedge C_{j}^{\prime \prime} \wedge C_{l}^{\prime \prime \prime}\right\}$ is a common cause of the correlation between $A_{m}$ and $B_{n}$ in the sense of (38)-(41) and suppose that $C_{i}^{\prime}, C_{j}^{\prime \prime}$ and $C_{l}^{\prime \prime \prime}$ are independent in the sense of (42). First, observe that (41) and (42) together entail that:

$$
p\left(a_{m} \wedge b_{n} \wedge C_{i}^{\prime} \wedge C_{j}^{\prime \prime} \wedge C_{l}^{\prime \prime \prime}\right)=p\left(a_{m} \wedge b_{n}\right) p\left(C_{i}^{\prime}\right) p\left(C_{j}^{\prime \prime}\right) p\left(C_{l}^{\prime \prime \prime}\right)
$$


Then $C_{j}^{\prime \prime}$ is a strong common cause. That is (43)-(46) hold:

$$
\begin{aligned}
& p\left(A_{m} \wedge B_{n} \mid a_{m} \wedge b_{n} \wedge C_{j}^{\prime \prime}\right) \quad=\quad \frac{p\left(A_{m} \wedge B_{n} \wedge a_{m} \wedge b_{n} \wedge C_{j}^{\prime \prime}\right)}{p\left(a_{m} \wedge b_{n} \wedge C_{j}^{\prime \prime}\right)} \\
& \stackrel{(55)}{=} \quad \frac{\sum_{i l} p\left(A_{m} \wedge B_{n} \mid a_{m} \wedge b_{n} \wedge C_{i}^{\prime} \wedge C_{j}^{\prime \prime} \wedge C_{l}^{\prime \prime \prime}\right) p\left(a_{m} \wedge b_{n}\right) p\left(C_{i}^{\prime}\right) p\left(C_{j}^{\prime \prime}\right) p\left(C_{l}^{\prime \prime \prime}\right)}{p\left(a_{m} \wedge b_{n}\right) p\left(C_{j}^{\prime \prime}\right)} \\
& \stackrel{(38)}{=} \quad \sum_{i l} p\left(A_{m} \mid a_{m} \wedge b_{n} \wedge C_{i}^{\prime} \wedge C_{j}^{\prime \prime} \wedge C_{l}^{\prime \prime \prime}\right) p\left(B_{n} \mid a_{m} \wedge b_{n} \wedge C_{i}^{\prime} \wedge C_{j}^{\prime \prime} \wedge C_{l}^{\prime \prime \prime}\right) p\left(C_{i}^{\prime}\right) p\left(C_{l}^{\prime \prime \prime}\right) \\
& \stackrel{(39)(40)}{=} \sum_{i l} p\left(A_{m} \mid a_{m} \wedge b_{n} \wedge C_{i}^{\prime} \wedge C_{j}^{\prime \prime}\right) p\left(B_{n} \mid a_{m} \wedge b_{n} \wedge C_{j}^{\prime \prime} \wedge C_{l}^{\prime \prime \prime}\right) p\left(C_{i}^{\prime}\right) p\left(C_{l}^{\prime \prime \prime}\right) \\
& \stackrel{(55)}{=} \quad p\left(A_{m} \mid a_{m} \wedge b_{n} \wedge C_{j}^{\prime \prime}\right) p\left(B_{n} \mid a_{m} \wedge b_{n} \wedge C_{j}^{\prime \prime}\right) \\
& p\left(A_{m} \mid a_{m} \wedge b_{n} \wedge C_{j}^{\prime \prime}\right) \quad=\quad \frac{p\left(A_{m} \wedge a_{m} \wedge b_{n} \wedge C_{j}^{\prime \prime}\right)}{p\left(a_{m} \wedge b_{n} \wedge C_{j}^{\prime \prime}\right)} \\
& \stackrel{(55)}{=} \quad \frac{\sum_{i l} p\left(A_{m} \mid a_{m} \wedge b_{n} \wedge C_{i}^{\prime} \wedge C_{j}^{\prime \prime} \wedge C_{l}^{\prime \prime \prime}\right) p\left(a_{m} \wedge b_{n}\right) p\left(C_{i}^{\prime}\right) p\left(C_{j}^{\prime \prime}\right) p\left(C_{l}^{\prime \prime \prime}\right)}{p\left(a_{m} \wedge b_{n}\right) p\left(C_{j}^{\prime \prime}\right)} \\
& \stackrel{(39)}{=} \quad \sum_{i l} p\left(A_{m} \mid a_{m} \wedge b_{n^{\prime}} \wedge C_{i}^{\prime} \wedge C_{j}^{\prime \prime} \wedge C_{l}^{\prime \prime \prime}\right) p\left(C_{i}^{\prime}\right) p\left(C_{l}^{\prime \prime \prime}\right) \\
& =\frac{\sum_{i l} p\left(A_{m} \mid a_{m} \wedge b_{n^{\prime}} \wedge C_{i}^{\prime} \wedge C_{j}^{\prime \prime} \wedge C_{l}^{\prime \prime \prime}\right) p\left(a_{m} \wedge b_{n^{\prime}}\right) p\left(C_{i}^{\prime}\right) p\left(C_{j}^{\prime \prime}\right) p\left(C_{l}^{\prime \prime \prime}\right)}{p\left(a_{m} \wedge b_{n^{\prime}}\right) p\left(C_{j}^{\prime \prime}\right)} \\
& \stackrel{(55)}{=} \quad \frac{p\left(A_{m} \wedge a_{m} \wedge b_{n^{\prime}} \wedge C_{j}^{\prime \prime}\right)}{p\left(a_{m} \wedge b_{n^{\prime}} \wedge C_{j}^{\prime \prime}\right)}=p\left(A_{m} \mid a_{m} \wedge b_{n^{\prime}} \wedge C_{j}^{\prime \prime}\right) \\
& p\left(B_{n} \mid a_{m} \wedge b_{n} \wedge C_{j}^{\prime \prime}\right) \quad=\quad \frac{p\left(B_{n} \wedge a_{m} \wedge b_{n} \wedge C_{j}^{\prime \prime}\right)}{p\left(a_{m} \wedge b_{n} \wedge C_{j}^{\prime \prime}\right)} \\
& \stackrel{(55)}{=} \quad \frac{\sum_{i l} p\left(B_{n} \mid a_{m} \wedge b_{n} \wedge C_{i}^{\prime} \wedge C_{j}^{\prime \prime} \wedge C_{l}^{\prime \prime \prime}\right) p\left(a_{m} \wedge b_{n}\right) p\left(C_{i}^{\prime}\right) p\left(C_{j}^{\prime \prime}\right) p\left(C_{l}^{\prime \prime \prime}\right)}{p\left(a_{m} \wedge b_{n}\right) p\left(C_{j}^{\prime \prime}\right)} \\
& \stackrel{(40)}{=} \quad \sum_{i l} p\left(B_{n} \mid a_{m^{\prime}} \wedge b_{n} \wedge C_{i}^{\prime} \wedge C_{j}^{\prime \prime} \wedge C_{l}^{\prime \prime \prime}\right) p\left(C_{i}^{\prime}\right) p\left(C_{l}^{\prime \prime \prime}\right) \\
& =\frac{\sum_{i l} p\left(B_{n} \mid a_{m^{\prime}} \wedge b_{n} \wedge C_{i}^{\prime} \wedge C_{j}^{\prime \prime} \wedge C_{l}^{\prime \prime \prime}\right) p\left(a_{m^{\prime}} \wedge b_{n}\right) p\left(C_{i}^{\prime}\right) p\left(C_{j}^{\prime \prime}\right) p\left(C_{l}^{\prime \prime \prime}\right)}{p\left(a_{m^{\prime}} \wedge b_{n}\right) p\left(C_{j}^{\prime \prime}\right)} \\
& \stackrel{(55)}{=} \quad \frac{p\left(B_{n} \wedge a_{m^{\prime}} \wedge b_{n} \wedge C_{j}^{\prime \prime}\right)}{p\left(a_{m^{\prime}} \wedge b_{n} \wedge C_{j}^{\prime \prime}\right)}=p\left(B_{n} \mid a_{m^{\prime}} \wedge b_{n} \wedge C_{j}^{\prime \prime}\right) \\
& p\left(a_{m} \wedge b_{n} \wedge C_{j}^{\prime \prime}\right) \quad=\quad \sum_{i l} p\left(a_{m} \wedge b_{n} \wedge C_{i}^{\prime} \wedge C_{j}^{\prime \prime} \wedge C_{l}^{\prime \prime \prime}\right) \\
& \stackrel{(55)}{=} \quad \sum_{i l} p\left(a_{m} \wedge b_{n} \wedge C_{i}^{\prime} \wedge C_{l}^{\prime \prime \prime}\right) p\left(C_{j}^{\prime \prime}\right)=p\left(a_{m} \wedge b_{n}\right) p\left(C_{j}^{\prime \prime}\right)
\end{aligned}
$$

where the numbers over the equation signs refer to the equation used at that step.

\section{References}

Bell, J.S., "Beables for quantum field theory," (TH-2053-CERN, presented at the Sixth GIFT Seminar, Jaca, 2-7 June 1975); reprinted in J. S. Bell, Speakable and Unspeakable in Quantum Mechanics, 
(Cambridge: Cambridge University Press, 2004, 52-62.).

Bell, J.S., "La nouvelle cuisine," in: J. Sarlemijn and P. Kroes (eds.), Between Science and Technology, Elsevier, (1990); reprinted in J. S. Bell, Speakable and Unspeakable in Quantum Mechanics, (Cambridge: Cambridge University Press, 2004, 232-248.).

J. Butterfield, "A Spacetime approach to the Bell inequality," in: J. Cushing and E. McMullin (eds.), Philosophical Consequences of Quantum Theory, Notre Dame, 114-144, (1989).

J. Butterfield, "Vacuum correlations and outcome independence in algebraic quantum field theory" in D. Greenberger and A. Zeilinger (eds.), Fundamental Problems in Quantum Theory, Annals of the New York Academy of Sciences, Proceedings of a conference in honour of John Wheeler, 768-785 (1995).

J. Butterfield, "Stochastic Einstein Locality Revisited," Brit. J. Phil. Sci., 58, 805-867, (2007).

C. Glymour, "Markov properties and quantum experiments," in W. Demopoulos and I. Pitowsky (eds.) Physical Theory and its Interpretation, (Springer, 117-126, 2006).

G. Grasshoff, S. Portmann and A. Wüthrich, "Minimal Assumption Derivation of a Bell-type Inequality," Brit. J. Phil. Sci., 56, 663-680 (2005).

J. Henson, "Comparing causality principles," Stud. Hist. Phil. Mod. Phys., 36, 519-543 (2005).

J. Henson, "Confounding causality principles: Comment on Rédei and San Pedro's "Distinguishing causality principles"," Stud. Hist. Phil. Mod. Phys., 44, 17-19 (2013a).

J. Henson, "Non-separability does not relieve the problem of Bell's theorem," Found. Phys., 43, 1008-1038 (2013b).

G. Hofer-Szabó, M. Rédei and L. E. Szabó, The Principle of the Common Cause, (Cambridge: Cambridge University Press, 2013).

G. Hofer-Szabó and P. Vecsernyés, "Reichenbach's Common Cause Principle in AQFT with locally finite degrees of freedom," Found. Phys., 42, 241-255 (2012a).

G. Hofer-Szabó and P. Vecsernyés, "Noncommuting local common causes for correlations violating the Clauser-Horne inequality," J. Math. Phys., 53, 12230 (2012b).

G. Hofer-Szabó and P. Vecsernyés, "Noncommutative Common Cause Principles in AQFT," J. Math. Phys., 54, 042301 (2013a).

G. Hofer-Szabó and P. Vecsernyés, "Bell inequality and common causal explanation in algebraic quantum field theory," Stud. Hist. Phil. Mod. Phys., 44 (4), 404-416 (2013b).

G. Hofer-Szabó and P. Vecsernyés, "On the concept of local causality in local classical and quantum theory," Stud. Hist. Phil. Mod. Phys. (submitted) (2014a).

G. Hofer-Szabó and P. Vecsernyés, "Bell's local causality for philosophers," Phil. Sci. (submitted) (2014b).

J. Jarrett, "On the Physical Significance of the Locality Conditions in Bell Arguments," Nous, 18, 569-589, (1984).

F. Muller and J. Butterfield, "Is Algebraic Lorentz-covariant Quantum Field Theory Stochastic Einstein Local?," Phil. Sci., 61, 457-74. (1994).

T. Norsen, "J.S. Bell's concept of local causality," Am. J. Phys, 79, 12, (2011). 
T. Placek and L. Wronski, "On infinite EPR-like correlations," Synthese., 167, 1-32 (2009).

H. Reichenbach, The Direction of Time, (University of California Press, Los Angeles, 1956).

M. Rédei, "Bell's inequalities, relativistic quantum field theory and the problem of hidden variables," Phil. Sci., 58, 628-638 (1991).

M. Rédei, "Reichenbach's Common Cause Principle and quantum field theory," Found. Phys., 27, 13091321 (1997).

M. Rédei and J. S. Summers, "Local primitive causality and the Common Cause Principle in quantum field theory," Found. Phys., 32, 335-355 (2002).

M. Rédei and I. San Pedro, "Distinguishing causality principles," Stud. Hist. Phil. Mod. Phys., 43, 84-89 (2012).

Shimony, A., "Events and processes in the quantum world," in: C. Isham and R. Penrose (eds.), Quantum Concepts in Space and Time, Oxford University Press, London/New York, 1986.

M. Suárez, "Interventions and Causality in Quantum Mechanics," Erkenntnis, 78/3, (2013).

Uffink, J., "The principle of the common cause faces the Bernstein paradox," Phil. Sci., 66 (3), 525 (1999).

Van Fraassen, B. C., "The Charybdis of Realism: Epistemological Implications of Bell's Inequality," Synthese, 52, 25-38 (1982). 